\title{
Solubility and dissolution enhancement of flurbiprofen by solid dispersion using hydrophilic carriers
}

\author{
Bhaskar Daravath ${ }^{1,2}$, Chella Naveen ${ }^{3}$, Sateesh Kumar Vemula ${ }^{4}$ and Rama Rao Tadikonda ${ }^{5 *}$
}

\begin{abstract}
${ }^{1}$ Department of Pharmaceutics, Max Institute of Pharmaceutical Sciences, Velugumatla, Wyra Road, Khammam, Telangana, India, ${ }^{2}$ Acharya Nagarjuna University, Nagarjuna Nagar, Guntur, Andhra Pradesh, India, ${ }^{3}$ Department of Pharmaceutics, National Institute of Pharmaceutical Education and Research, Hyderabad, India, ${ }^{4}$ Department of Pharmaceutics, Chaitanya College of Pharmacy Education and Research, Hanamkonda, AP, India, ${ }^{5}$ Department of Pharmaceutics, Avanthi Institute of Pharmaceutical Sciences, Hayath Nagar, Ranga Reddy (D), Hyderabad, Telangana, India
\end{abstract}

\begin{abstract}
The intent of the current work is to study the effect of polyethylene glycol 8000 and polyethylene glycol 10000 as hydrophilic carriers on dissolution behaviour of flurbiprofen. In the present study, solvent evaporation method was used to prepare flurbiprofen solid dispersions and evaluated for physicochemical properties, drug-carrier compatibility studies and dissolution behaviour of drug. Solubility studies showed more solubility in higher $\mathrm{pH}$ values and formulations SD4 and SD8 were selected to prepare the fast dissolving tablets. FTIR and DSC study showed no interaction and drug was dispersed molecularly in hydrophilic carrier. XRD studies revealed that there was change in the crystallinity of the drug. The results of In vitro studies showed SD8 formulation confer significant improvement $(\mathrm{p}<0.05)$ in drug release, $\mathrm{Q}_{20}$ was $99.08 \pm 1.35 \%$ compared to conventional and marketed tablets $(47.31 \pm 0.74 \%$ and $56.86 \pm 1.91 \%$ ). The mean dissolution time (MDT) was reduced to $8.79 \mathrm{~min}$ compared to conventional and marketed tablets (25.76 and $22.22 \mathrm{~min}$.) indicating faster drug release. The DE (\% dissolution efficiency) was increased by 2.5 folds $(61.63 \%)$ compared to conventional tablets $(23.71 \%)$. From the results, it is evident that polyethylene glycol solid dispersions in less carrier ratio may enhance the solubility and there by improve the dissolution rate of flurbiprofen.
\end{abstract}

Keywords: Polyethylene glycol/effects. Flurbiprofen/solubility. Flurbiprofen/dissolution rate. Drugcarrier compatibility/study. Dissolution efficiency.

\section{INTRODUCTION}

In the pharmaceutical industries, formulation scientists has a major challenge in the formulation of orally administered solid dosage forms is solubility enhancement and improvement of dissolution of poor water soluble drugs (Horter, Dressman, 2001).Solid dispersion technologies enhance the solubility and dissolution of drugs and thereby improve the oral bioavailability of poorly water soluble drugs (Patil, Gaikwad, 2009). Thus, drug's acceptability and bioavailability will depends on enhancement of solubility and dissolution that reduces dose required for showing fast onset of action (Chauhan, Shimpi, Paradkar, 2005).

\footnotetext{
*Correspondence: R. R. Tadikonda. Department of Pharmaceutics. Avanthi Institute of Pharmaceutical Sciences. Hayath Nagar, Ranga Reddy (D), Hyderabad 500090. Telangana, India. Email: tramarao2014@gmail.com
}

Solid dispersions (SDs) are molecular dispersions of drug in the carriers and they were formulated by different methods, important are solvent evaporation and fusion (melt) method (Vippagunta, Maul, Tallavajhala, 2002). SD is a technique, in which drug is obtained as fine particles by dissolving the carriers in an aqueous fluids. It is a simple and flexible formulation process of obtaining solid dispersions without using toxic substances and enhances solubility and dissolution rate of drug (Bikiaris, 2011).

Flurbiprofen (FPN) is non-steroidal anti-inflammatory drug that belongs to BCS class II because of its poor water solubility (Veerareddy, Vemula, 2012). Different molecular weights of polyethylene glycols (PEG8000 and PEG10000) were used for the preparation of SDs. In this work, solvent evaporation method was employed for formulating FPN solid dispersions utilizing PEGs as carrier.

The reported literatures for solid dispersions arenisoldipine (El-Maghraby, Elsergany, 2014), simvastatin 
(Bolourchian, Mahboobian, Dadashzadeh, 2013), lycopene (Faisal et al., 2013), diclofenac sodium (Cwiertnia, 2013), gliclazide (Biswal, Sahoo, Murthy, 2009) and ritonavir (Sinha, Ali, Baboota, 2010). The need of present study is to develop the solid dispersions in less carrier ratio with improved results (Ozkanet al., 2000). In the current work, we prepared FPNSDs to increase solubility and dissolution of the drug using PEGs as carrier.

\section{MATERIAL AND METHODS}

\section{Material}

Flurbiprofen was kindly gifted FDC Ltd., (Mumbai, India). PEG 8000 and PEG 10000 were gift samples from Central Drug House Pvt. Ltd., (Delhi, India). Crospovidone and Spray-dried lactose were obtained from Matrix laboratories Ltd., (Hyderabad, India). All other chemicals and reagents were purchased from S.D. Fine Chemicals, (Mumbai, India).

\section{Phase solubility studies of FPN}

An excess amount of FPN was taken into a flask containing aqueous solution $(0,5,10,15,20,25$ and $30 \%$ w/v)of PEG 8000 and PEG 10000 separately (Suresh et $a l ., 2013)$.The samples were continuously stirred on a stirrer for $48 \mathrm{~h}$ at room temperature. The samples were filtered by using $0.45 \mu \mathrm{m}$ filter. Then clear filtrates were appropriately diluted and drug concentration was analysed byUV spectrophotometer at $247 \mathrm{~nm}$.

The solubilisation of drug in an aqueous solution was explained by Gibbs free energy of transfer $\left(\Delta \mathrm{G}^{\mathrm{o}}{ }_{\mathrm{tr}}\right)$ and determined by equation given below.

$$
\left(\Delta \mathrm{G}_{\mathrm{tr}}^{\mathrm{o}}\right)=-2.303 \mathrm{RT} \log \mathrm{S}_{\mathrm{o}} / \mathrm{S}_{\mathrm{s}}
$$

TABLE I - Formulation of FPN solid dispersions using PEGs where, $\mathrm{S}_{\mathrm{o}} / \mathrm{S}_{\mathrm{s}}$ was FPN molar solubility ratio in PEGs solutions to that of water.

\section{Preparation of FPN solid dispersions}

Solvent evaporation method was employed to prepare FPNSD susing PEG8000 and PEG10000 as continuous phase in different weight ratios (Table I). PEGs and drug were dissolved in a round bottom flask containing 10 mLof ethanol. At a temperature not exceeding 45 ${ }^{\circ} \mathrm{C}$, the solvent was evaporated in a vacuum oven. The resultant solid dispersions were grounded in a mortar with the help of pestle, kept in a vial and were stored in desiccators till further use (Christian, Jennifer, 2000).

\section{Solubility studies}

Solubility studies were performed for prepared SDs in various solvent systems ( $0.1 \mathrm{~N}$ hydrochloric acid, distilled water and $\mathrm{pH} 7.2$ phosphate buffer). Excessive quantities of SDs were placed into a flask containing 10 $\mathrm{ml}$ of each solvent. The flask was sonicated at $25^{\circ} \mathrm{C}$ for 1 $\mathrm{h}$, stirred and agitated for 2 days at $25^{\circ} \mathrm{C}$. The suspension was filtered using a $0.45 \mu \mathrm{m}$ filter, diluted suitably and spectrophotometrically (UV-3200, LabIndia, Mumbai, India) analyzed at $247 \mathrm{~nm}$.

\section{Flow properties of powder}

Angle of repose and Carr's compressibility index were used to determine the powder flow properties. Angle of repose $(\theta)$ was measured by fixed funnel method and it was determined by given formula:

$$
\operatorname{Tan} \theta=\frac{\mathrm{h}}{\mathrm{r}}
$$

\begin{tabular}{lcccc}
\hline \multirow{2}{*}{ Formulation Code } & \multicolumn{4}{c}{ Excipients (mg) } \\
\cline { 2 - 5 } & Flurbiprofen & PEG 8000 & PEG 10000 & FPN:PEG ratio \\
\hline SD1 & 50 & 25 & - & $1: 0.5$ \\
SD2 & 50 & 50 & - & $1: 1$ \\
SD3 & 50 & 100 & - & $1: 2$ \\
SD4 & 50 & 200 & - & $1: 4$ \\
SD5 & 50 & - & 25 & $1: 0.5$ \\
SD6 & 50 & - & 50 & $1: 1$ \\
SD7 & 50 & - & 100 & $1: 2$ \\
SD8 & 50 & - & 200 & $1: 4$ \\
\hline
\end{tabular}


where ' $\theta$ ' was angle of repose, ' $h$ ' and ' $r$ ' were height and radius of the cone of powder.

The tendency of a powder to be compressed can measured by Carr's index and determined using given below formula:

$$
\text { Carr's Index }=\frac{\left[\left(\rho_{\mathrm{P}}-\rho_{\mathrm{b}}\right)\right]}{\rho_{\mathrm{P}}} \times 100
$$

where ' $\rho_{\mathrm{b}}$ ' and ' $\rho_{\mathrm{p}}$ ' were bulk density and tapped density respectively.

\section{Preparation of tablets}

From the results of above studies, SD4 and SD8 formulations were prepared in oro dispersible tablets (ODTs) form (Table II). SDs equivalent to $50 \mathrm{mg}$ of FPN and all other ingredients were sieved (\# 60). Except lubricant and glidant, powder blend was mixed for $5 \mathrm{~min}$. The powder blend was then lubricated for 2-5 min and compressed directly using rotary compression machine (CMD-3, Cadmach, Ahmedabad, India). In this way, conventional tablets were also prepared using $50 \mathrm{mg}$ of pure FPN.

\section{Characterization of tablets}

The ODTs were characterized by different evaluation tests like weight variation, friability, hardness, disintegration, wetting time and drug content. All the tests were performed as per the procedure described in our previous work (Bhaskar, Rama, Sateesh, 2015).

\section{In-vitro dissolution studies}

The in-vitro dissolution studies of various FPN formulation (SD4, SD8, marketed and conventional formulations) was performed in USP Type II dissolution apparatus (TDL - 08L, Electrolab, Mumbai, India) using $900 \mathrm{~mL}$ of phosphate buffer $(\mathrm{pH}$ 7.2) rotated at $50 \mathrm{rpm}$ and maintained at $37.0 \pm 0.5^{\circ} \mathrm{C}$. Aliquots of $5 \mathrm{ml}$ were withdrawn at pre-determined time intervals and the same amount of fresh dissolution media was replaced. The samples were analyzed by UV spectrophotometer at 247 $\mathrm{nm}$ after filtration through $0.45 \mu \mathrm{m}$ filter and appropriate dilution.

\section{Dissolution data treatment}

Cumulative \% drug release in 20 min $\left(\mathrm{Q}_{20}\right)$, mean dissolution time (MDT), dissolution efficiency ( $\% \mathrm{DE})$ and relative dissolution rate (RDR) were determined to compare the optimized SDs with marketed tablets and conventional formulation of FPN as discussed in our previous article (Chella et al., 2016).

\section{Drug-carrier compatibility studies}

Drug-carrier interaction studies were performed using Fourier Transform Infrared Spectroscopy (FTIR), Differential Scanning Calorimetry (DSC) and powder X ray diffraction (PXRD) studies. FTIR spectra of FPN, PEG and optimized SD were recorded on IR spectrophotometer (Shimadzu, Japan)in the range of 400 to $4000 \mathrm{~cm}^{-1}$ using pellets compacted by mixing approximately $5 \mathrm{mg}$ of samples $100 \mathrm{mg}$ of potassium bromide $(\mathrm{KBr})$ under vacuum. The DSC thermograms of above said samples were recorded by placing approximately $5-10 \mathrm{mg}$ sample in sealed aluminium pan with small openings on a differential scanning colorimetry(Shimadzu Corp., Japan), at a heating rate of $5{ }^{\circ} \mathrm{C}$ per min and a temperature from 0 to $400{ }^{\circ} \mathrm{C}$ under a flow of nitrogen gas. X-ray diffractometer (Siemens D5000, Texas, USA) was used to record the diffractograms. FPN, PEG 10000 and SD8 were exposed to $\mathrm{Cu}$ radiation

TABLE II - Composition of selected FPN SDs tablets

\begin{tabular}{lccc}
\hline \multirow{2}{*}{ Formulation composition } & \multicolumn{3}{c}{ Quantity in mg } \\
\cline { 2 - 4 } & SD4 & SD8 & Conventional \\
\hline FPNSDs equivalent to 50 mg of FPN & 250 & 250 & - \\
Plain FPN & - & - & 50 \\
Crospovidone & 15 & 15 & 15 \\
Spry-dried lactose & 26 & 26 & 226 \\
Magnesium stearate & 3 & 3 & 3 \\
Talc & 6 & 6 & 6 \\
\hline Total weight of tablet & 300 & 300 & 300 \\
\hline
\end{tabular}


of wavelength $1.540 \AA, 40 \mathrm{KV}$ voltage $30 \mathrm{~mA}$ current and analysed over the $2 \theta$ range of $2^{\circ}$ to $50^{\circ}$.

\section{Stability studies}

Stability of optimized formulation (SD8) was performed as mentioned in the literature (Mathews, 1999). The optimized ODTs were packed in aluminum paper and placed in the stability chamber for 6 months at $75 \pm 5 \% \mathrm{RH}$ and $40 \pm 2^{\circ}$. After completion of the storage period, in-vitro dissolution and assay were performed for collected samples. Paired t-test was performed to check the significant difference at $p=0.05$. The similarity between before and after storage was determined by similarity index (F2) which indicates stability of optimized formulation.

\section{RESULTS AND DISCUSSIONS}

\section{Results}

\section{Phase solubility studies}

The results of phase solubility study presented in Table III indicate increase in FPN solubility linearly $\left(\mathrm{R}^{2}\right.$ $=0.970$ with PEG 8000 and $\mathrm{R}^{2}=0.973$ with PEG 10000) as the concentration of PEGs was increased. The phase solubility diagram is shown in Figures $1 \mathrm{a}$ and $1 \mathrm{~b}$.

\section{Solubility studies of FPN SDs}

Solubility studies of solid dispersion were performed in various solvent systems and the results are shown in Table IV. Solubility of FPN is higher in $7.2 \mathrm{pH}$ phosphate buffer compared to $0.1 \mathrm{~N} \mathrm{HCl}$ and distilled water whereas, SDs showed more or less equal solubility in both water and phosphate buffer. The solubility of
SDs increased with increase in concentration of carrier in both cases (PEG 8000 and PEG 10000). However,
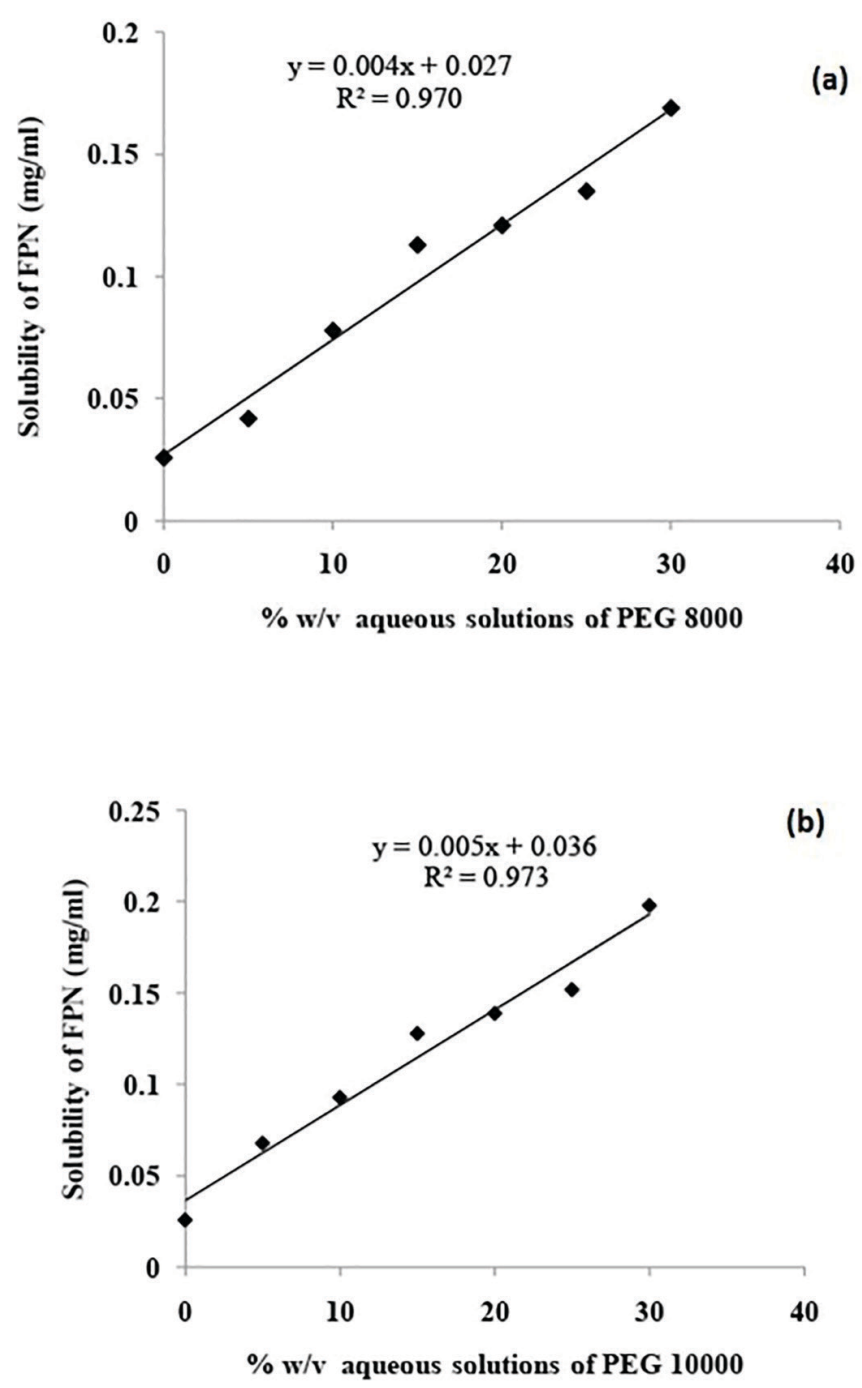

FIGURE 1 - Phase solubility curves of FPN in aqueous solution of PEGs at $25^{\circ} \mathrm{C}(\mathrm{n}=3)$ [(a) in PEG 8000, (b) in PEG 10000].

TABLE III - Phase Solubility data of FPN in different concentrations of PEG 8000 and PEG 10000 aqueous solutions (Mean \pm SD, n=3)

\begin{tabular}{lcccc}
\hline $\begin{array}{l}\text { Concentration of } \\
\text { carrier solution } \\
\mathbf{( \% , ~ w / v ) ~}\end{array}$ & $\begin{array}{c}\text { Solubility of FPN } \\
(\mathbf{m g} / \mathbf{m l})\end{array}$ & $\begin{array}{c}\Delta \mathbf{G}^{\mathbf{0}} \mathbf{t r} \\
(\mathbf{K j} / \mathbf{m o l})\end{array}$ & $\begin{array}{c}\text { Solubility of FPN } \\
(\mathbf{m g} / \mathbf{m l})\end{array}$ & $\begin{array}{c}\Delta \mathbf{G}^{\circ} \mathbf{t r} \\
(\mathbf{K j} / \mathbf{m o l})\end{array}$ \\
\hline 0 & $0.026 \pm 0.0035$ & - & $0.026 \pm 0.0052$ & - \\
5 & $0.068 \pm 0.0024$ & $-1.19 \pm 0.153$ & $0.068 \pm 0.0072$ & $-2.38 \pm 0.134$ \\
10 & $0.093 \pm 0.0071$ & $-2.72 \pm 0.182$ & $0.093 \pm 0.0031$ & $-3.16 \pm 0.185$ \\
15 & $0.098 \pm 0.0014$ & $-3.64 \pm 0.196$ & $0.098 \pm 0.0068$ & $-3.95 \pm 0.191$ \\
20 & $0.129 \pm 0.0029$ & $-3.81 \pm 0.175$ & $0.129 \pm 0.0093$ & $-4.15 \pm 0.142$ \\
25 & $0.142 \pm 0.0083$ & $-4.08 \pm 0.131$ & $0.142 \pm 0.0084$ & $-4.38 \pm 0.167$ \\
30 & $0.175 \pm 0.0062$ & $-4.64 \pm 0.173$ & $0.175 \pm 0.0042$ & $-5.03 \pm 0.125$ \\
\hline
\end{tabular}


TABLE IV- Solubility of FPN and its SDs (Mean $\pm S D ; \mathrm{n}=6$ )

\begin{tabular}{lccc}
\hline \multirow{2}{*}{ Formulation Code } & \multicolumn{3}{c}{ FPN solubility (mg/ml) } \\
\cline { 2 - 4 } & $\mathbf{0 . 1} \mathbf{~ N ~ H C l}$ & Distilled Water & $\mathbf{7 . 2} \mathbf{~ p H ~ P h o s p h a t e ~ B u f f e r ~}$ \\
\hline Pure FPN & $0.014 \pm 0.03$ & $0.026 \pm 0.08$ & $0.098 \pm 0.01$ \\
SD1 & $0.113 \pm 0.02$ & $0.257 \pm 0.02$ & $0.298 \pm 0.07$ \\
SD2 & $0.126 \pm 0.04$ & $0.338 \pm 0.06$ & $0.349 \pm 0.02$ \\
SD3 & $0.136 \pm 0.04$ & $0.412 \pm 0.04$ & $0.425 \pm 0.06$ \\
SD4 & $0.143 \pm 0.01$ & $0.493 \pm 0.06$ & $0.526 \pm 0.07$ \\
SD5 & $0.102 \pm 0.02$ & $0.294 \pm 0.03$ & $0.324 \pm 0.08$ \\
SD6 & $0.123 \pm 0.05$ & $0.349 \pm 0.06$ & $0.387 \pm 0.06$ \\
SD7 & $0.138 \pm 0.08$ & $0.414 \pm 0.01$ & $0.473 \pm 0.04$ \\
SD8 & $0.156 \pm 0.01$ & $0.517 \pm 0.06$ & $0.561 \pm 0.02$ \\
\hline
\end{tabular}

SDs prepared with PEG10000 showed higher solubility in all concentrations compared to SDs prepared with PEG8000. Among all formulations, SD8 showed highest solubility in phosphate buffer $\mathrm{pH} 7.2$ i.e., $0.561 \pm 0.02 \mathrm{mg} / \mathrm{mL}$.

\section{Flow properties of powder}

Angle of repose and Carr's compressibility index of the pure FPN and optimised formulations (SD4 and SD8) is reported in Table $\mathrm{V}$. The angle of repose values lower than $30^{\circ}$ and Carr's compressibility index less than 18 for two formulations indicates good flow properties for pure FPN and SDs.

\section{Characterisation of tablets}

The results of the tests performed were within the pharmacopoeial limits (Table VI). For the weight variation test, all the tablets were within $5 \%$ of average weight of tablets. The friability and hardness were found near to be $0.35 \%$ and $3.1 \mathrm{~kg} / \mathrm{cm}^{2}$ for optimized SD 8 formulation. The disintegration and wetting time of the optimized tablet were found to be $121 \mathrm{sec}$ and $26 \mathrm{sec}$ respectively, indicating rapid disintegration of optimised tablets. Tablets contain $99.24 \%$ of FPN.
TABLE V- Flow properties of FPN and formulations (Mean \pm $\mathrm{SD}, \mathrm{n}=3$ )

\begin{tabular}{lcc}
\hline $\begin{array}{l}\text { Formulation } \\
\text { Code }\end{array}$ & $\begin{array}{c}\text { Angle of } \\
\text { Repose ( }\end{array}$ & $\begin{array}{c}\text { Carr's } \\
\text { Index (\%) }\end{array}$ \\
\hline SD4 & $28.24 \pm 0.57$ & $15.07 \pm 0.36$ \\
SD8 & $27.06 \pm 0.79$ & $15.32 \pm 0.25$ \\
Conventional & $29.72 \pm 3.15$ & $17.02 \pm 0.42$ \\
\hline
\end{tabular}

\section{In vitro dissolution studies}

The ODTs containing PEG 10000 SD showed higher release of FPN compared with to ODTs containing PEG8000 SD, marketed and conventional tablets of plain FPN. The optimized formulation (SD8) containing PEG 10000 showed significantly improved $(\mathrm{p}<0.05)$ and faster drug release $(99.08 \pm 1.35 \%$ in $20 \mathrm{~min})$ compared with formulation SD4 containing PEG $8000(93.67 \pm 1.12 \%$ in $20 \mathrm{~min})$, marketed tablet (56.86 $\pm 1.91 \%)$ and conventional tablet $(47.31 \pm 0.74 \%$ in $20 \mathrm{~min})$. The release pattern of FPN from various formulations is shown in Figure 2.

\section{Treatment of dissolution data}

The $\%$ FPN release from optimized formulation

TABLE VI - Physical evaluation of FPN tablets (Mean $\pm S D, * n=20 ; \uparrow n=6 ;+n=3$ )

\begin{tabular}{|c|c|c|c|c|c|c|}
\hline Formulation & $\begin{array}{c}\text { Weight } \\
\text { variation* }(\mathrm{mg})\end{array}$ & $\begin{array}{c}\text { Friability } \\
(\%)\end{array}$ & $\begin{array}{c}\text { Hardness } \dagger \\
\left(\mathrm{Kg} / \mathrm{cm}^{2}\right)\end{array}$ & $\begin{array}{c}\text { Disintegration } \\
\text { time } \$(\mathrm{sec})\end{array}$ & $\begin{array}{l}\text { Wetting time: } \\
\text { (sec) }\end{array}$ & $\begin{array}{c}\text { Drug content } \\
(\%)\end{array}$ \\
\hline SD4 & $301.51 \pm 1.18$ & $0.38 \pm 0.053$ & $3.0 \pm 0.27$ & $120 \pm 4$ & $28 \pm 6$ & $98.92 \pm 1.53$ \\
\hline SD8 & $300.12 \pm 1.72$ & $0.35 \pm 0.072$ & $3.1 \pm 0.43$ & $121 \pm 3$ & $26 \pm 3$ & $99.24 \pm 1.26$ \\
\hline Conventional & $301.68 \pm 1.13$ & $0.27 \pm 0.046$ & $3.1 \pm 0.14$ & $248 \pm 4$ & $45 \pm 2$ & $97.36 \pm 1.47$ \\
\hline
\end{tabular}



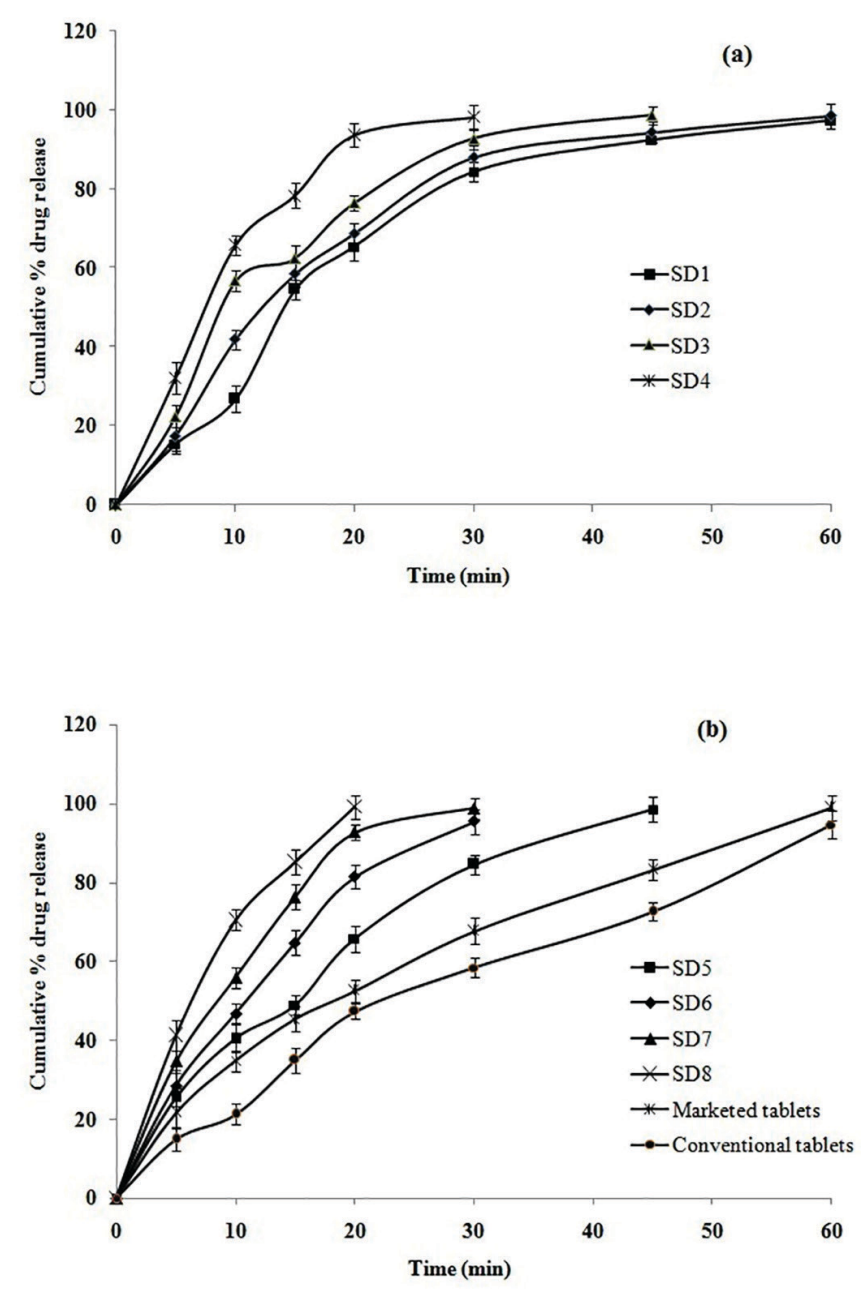

FIGURE 2 - Cumulative\% drug release of FPN from various formulations.

(SD8) was significantly higher compared to conventional tablets in $20 \mathrm{~min}$. DE(\%), MDT, and RDR were determined. $\mathrm{DE}(\%)$ was found to be $61.63 \%$ and MDT was reduced to 8.79 min compared with conventional tablet $(23.71 \pm 1.46 \%$ and $25.76 \mathrm{~min}$ respectively). The relative dissolution rate of optimized SD8 formulation was found to be 2.09.

\section{Drug-carrier compatibility studies}

FT IR spectra of pure FPN, PEG 10000 and optimised formulation SD8 is shown in Figure 3.FTIR spectra of FPN exhibits prominent peaks at $2998.43 \mathrm{~cm}^{-1}$ (C-H stretching vibration), $1463.51 \mathrm{~cm}^{-1}$ (C-H bending vibration), $1721.37 \mathrm{~cm}^{-1}(\mathrm{C}=\mathrm{O}$ carbonyl stretch of acid), $1576.24 \mathrm{~cm}^{-1}(\mathrm{C}=\mathrm{C}$ stretching of aromatic ring $)$ and $1236.63 \mathrm{~cm}^{-1}$ (C-F stretching). Presence of all the principal peaks of FPN in SD8 formulation and absence of new peaks indicated that interaction was not present between the FPN and PEG 10000.
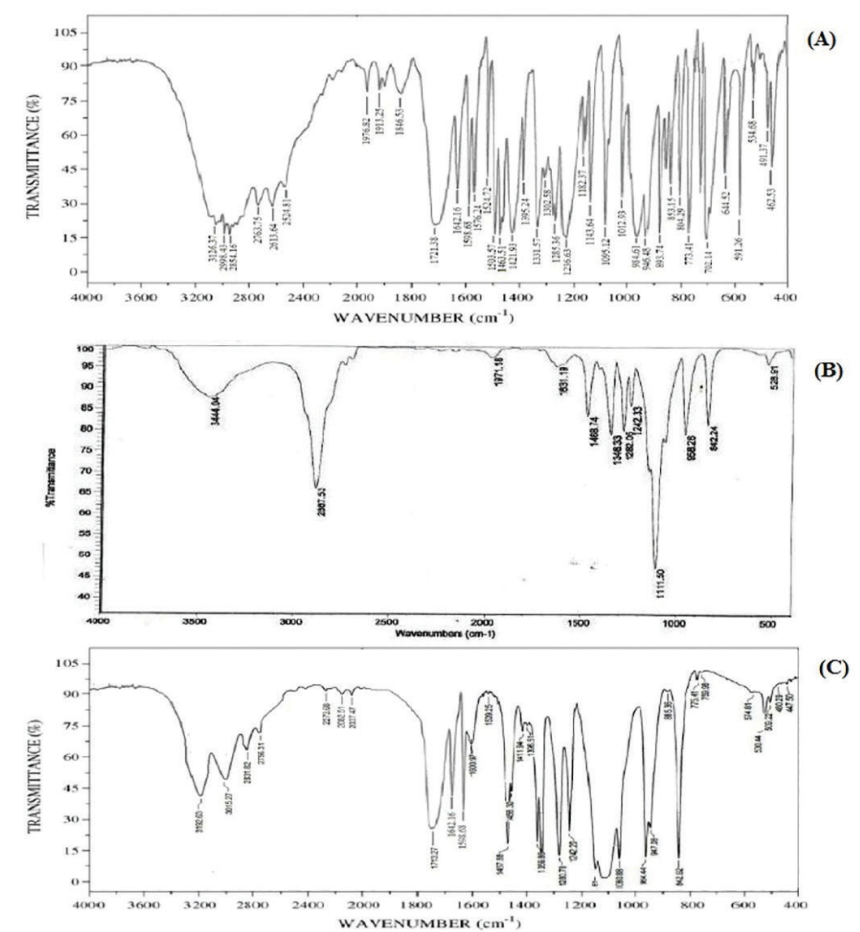

FIGURE 3 - FTIR spectra of A) FPN B) PEG 10000 C) SD8 formulation.

DSC thermograms obtained for pure FPN, PEG 10000 and SD8 solid dispersion are presented in Figure 4. The thermogram of FPN showed a sharp endothermic peak at $113.4^{\circ}$ characteristic of its melting point, whereas thermogram of the SD8 formulation showed broad endothermic peak of drug, indicating that FPN was molecularly dispersed in carrier.

The X-ray diffractograms of pure FPN, PEG 10000

TABLE VII - Dissolution parameters of FPN from SD4, SD8, marketed and conventional tablets $(\mathrm{Mean} \pm \mathrm{SD}, \mathrm{n}=3)$

\begin{tabular}{lcccc}
\hline Formulation & $\left(\mathbf{Q}_{\mathbf{2 0}}\right)$ & DE $\mathbf{( \% )}$ & MDT $(\mathbf{m i n})$ & RDR \\
\hline SD4 & $93.67 \pm 1.76$ & $55.74 \pm 1.31$ & $8.87 \pm 0.32$ & $1.98 \pm 0.014$ \\
SD8 & $99.08 \pm 1.35$ & $61.63 \pm 1.62$ & $8.79 \pm 0.14$ & $2.09 \pm 0.032$ \\
Marketed & $56.86 \pm 1.91$ & $31.97 \pm 1.25$ & $22.22 \pm 0.17$ & $1.11 \pm 0.018$ \\
Conventional & $47.31 \pm 0.74$ & $23.71 \pm 1.46$ & $25.76 \pm 0.26$ & - \\
\hline
\end{tabular}



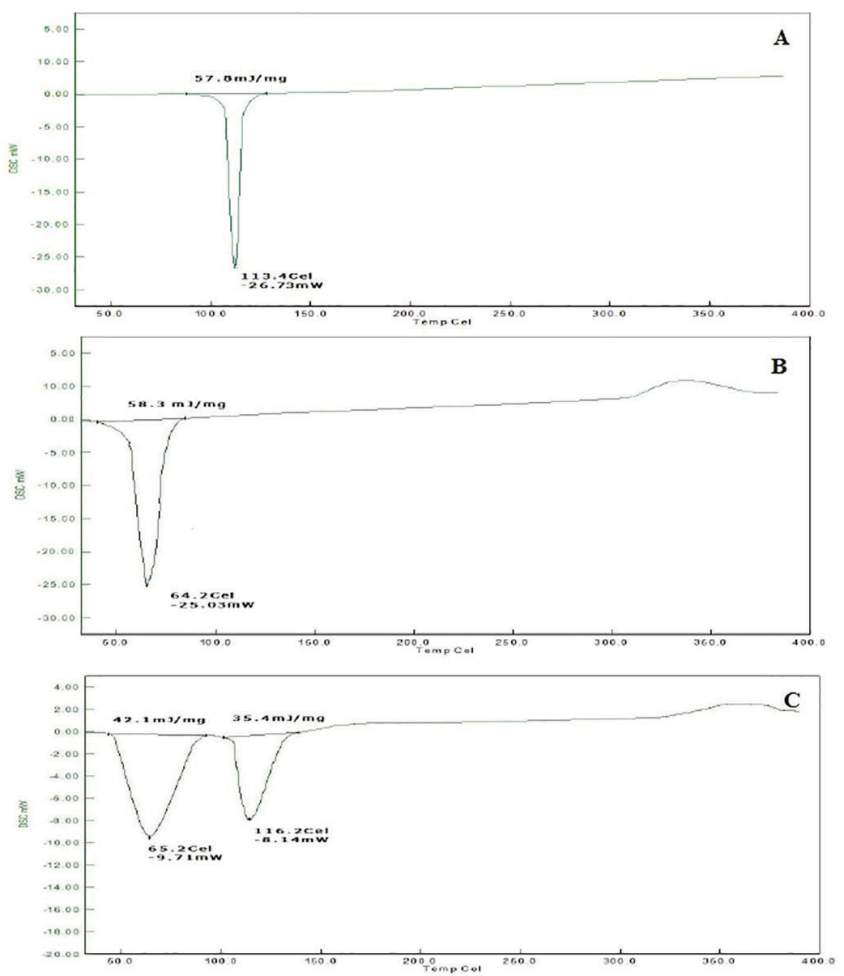

FIGURE 4 - DSC thermograms of A) FPN B) PEG 10000 C) SD8 formulation.

and SD8 solid dispersion are presented in Figure 5. XRD patterns of FPN shows sharp intense peaks notably at $2 \theta$ diffraction angles of $7^{\circ}, 11^{\circ}, 16^{\circ}, 17^{\circ}, 21^{\circ}, 22^{\circ}$, and $24^{\circ}$ indicating FPN was in crystalline state. The decrease or disappearance of peaks intensity in SD8 formulation indicates that FPN may have undergone solid state transition to amorphous form or crystallinity was reduced.

\section{Stability studies}

Stability of the optimised formulation (SD8) was evaluated at accelerated condition. In-vitro dissolution studies and assay were performed after the completion of storage period. There was no significant variation i.e.,

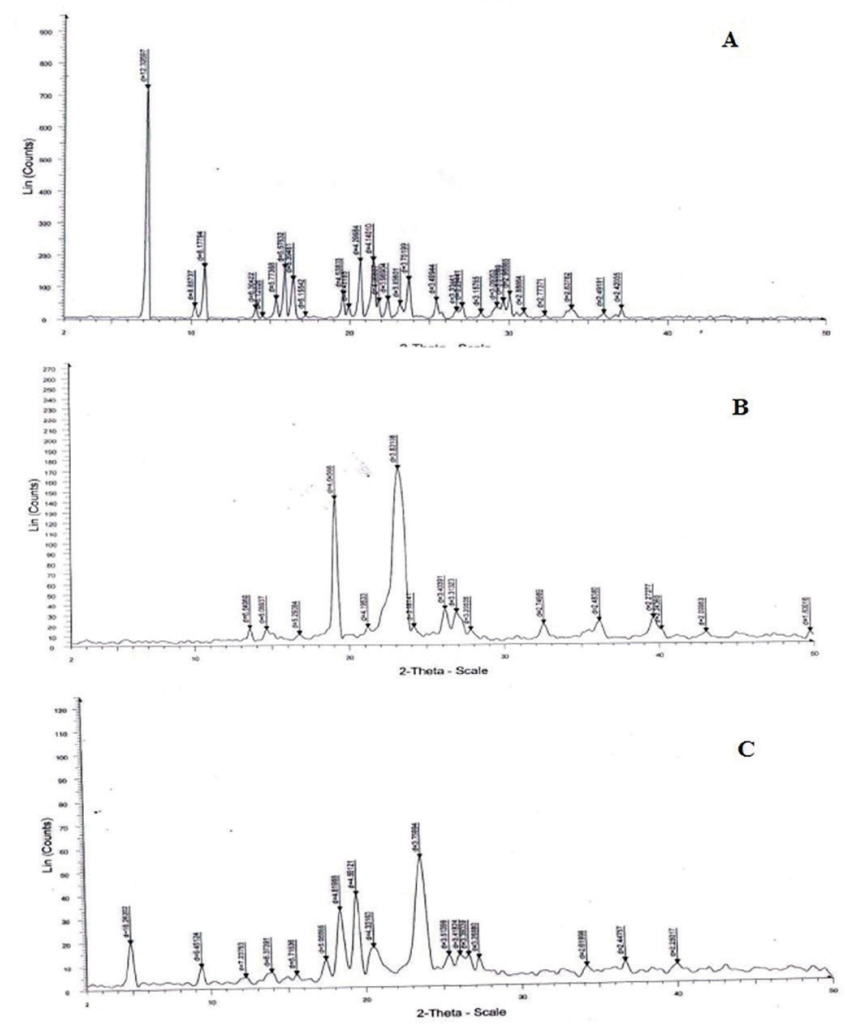

FIGURE 5 - XRD patterns of A) FPN B) PEG 10000 C) SD8 formulation.

$\mathrm{p}>0.05$ in concentration of drug (Table VIII) determined by using paired t-test. Similarity index value was found to be 89.59 .

\section{DISCUSSION}

The intent of current work is to study the role of hydrophilic carriers (PEG 8000 and PEG 10000) in improving the solubility and dissolution rate of FPN at different concentrations. FPN SDs were prepared by solvent evaporation method using PEG 8000 and PEG 10000 at four different concentrations of FPN to PEG and optimised SD was compressed into ODTs.

TABLE VIII - Stability study data of ODTs containing SD8 $($ Mean \pm SD, $n=3)$

\begin{tabular}{lcccc}
\hline Time (min) & Before storage & After 6 months Storage & $\boldsymbol{t}$-test at 0.05 LS & Similarity Factor (F2) \\
\hline 0 & $0.00 \pm 0.00$ & $0.00 \pm 0.00$ & & \\
5 & $41.28 \pm 1.71$ & $40.12 \pm 1.38$ & & \\
10 & $70.49 \pm 1.16$ & $69.27 \pm 1.12$ & Not Significant & 89.59 \\
15 & $85.22 \pm 1.08$ & $83.84 \pm 1.43$ & & \\
20 & $99.08 \pm 1.43$ & $97.78 \pm 1.25$ & & \\
\hline \% Assay & $99.34 \pm 1.26$ & $98.64 \pm 1.23$ & Not Significant & -- \\
\hline
\end{tabular}


Finally, solubility and dissolution rate of FPN from ODT containing SD was compared with that of marketed formulations and conventional tablets prepared with pure FPN.

Linear increment in solubility of FPN was observed with increase in concentration of PEGs. As the calculated slopes were less than one, the phase solubility diagram follows an $\mathrm{A}_{\mathrm{L}}$-type of solubility diagram (Suresh et al., 2013). Negative values of $\Delta \mathrm{G}_{\mathrm{tr}}^{\circ}$ (Gibbs free energy transfer) indicated that spontaneous solubilisation of FPN and it was decreased as the concentration of PEGs increases, indicating more favourable reaction with increase in concentration of PEGs.

Solid dispersions of FPN were prepared using various concentrations of PEG 8000 and PEG 10000 separately. In presence of PEG the solubility was increased up to drug to carrier ratio 1:4. Further increase in ratio to 1:5 and 1:6 did not show any significant improvement in the solubility. Hence, the formulations up to 1:4 ratios were included in the present study. Similar types of results were reported by Chauhan et. al., (Chauhan, Shimpi, Paradkar, 2005). The solubility of FPN from SDs prepared with PEG 10000 showed higher solubility compared with PEG 8000. Among all formulations, SD8 showed higher solubility in phosphate buffer $\mathrm{pH}$ 7.2.

Angle of repose and Carr's compressibility index were determined to find out the flow behaviour of powder. The angle of repose $\left(<30^{\circ}\right)$ and Carr's compressibility index $(<18)$ values indicated that the powder blend has good flow property. Then FPN solid dispersions (SD1 to SD8) were compressed into orodispersible tablets (ODTs) based on the solubility data. Prepared ODTs were evaluated for various pharmacopoeial and other general tests like weight variation, hardness, friability, disintegration time, wetting time and drug content. All the tests were within the pharmacopoeial limits.

In vitro dissolution studies showed, ODTs containing PEG 10000 SDs showed higher release of FPN compared with ODTs containing PEG8000, marketed and conventional tablets. All the formulations containing FPN in the form of SDs showed higher drug release compared to marketed tablets and conventional tablets containing plain FPN. Formulation SD5 (with FPN to drug PEG 10000 ratio $1: 1$ ) showed complete drug release in 45 min. SD6 and SD7 formulations with FPN to drug PEG 10000 ratio $1: 2$ and $1: 3$ respectively showed complete drug release in $30 \mathrm{~min}$. These values were higher than the results reported by Dong et al. (2011), who reported only $70 \%$ drug release in $60 \mathrm{~min}$ (Dong et al., 2011). Whereas, Formulation SD8 containing PEG 10000 of 1:4 ratio showed faster drug release $(99.08 \pm 1.35 \%$ in $20 \mathrm{~min})$ with significantly improved dissolution $(\mathrm{p}<0.05)$ compared with all other formulations (Figure 2).As the selected drug belongs to NSAID that needs to produce faster action we selected SD8 as final optimised formulation.

From in vitro dissolution studies it was evident that enhancement in dissolution rate was observed with tablets containing PEG 10000 compared with PEG 8000. It may be due to greater carrier solubilization effect, increased dispersibility and wettability of drug, increased dissolution in hydrophilic carrier, change in state of drug (may be amorphous form), decrease in crystallinity and/or a combination of the above said mechanisms (Dordunoo, Ford, Rubisteins, 1991).

The \% flurbiprofen release $\left(\mathrm{Q}_{20}\right)$ from formulation SD8 was significantly higher compared to conventional tablets in $20 \mathrm{~min}$. Enhancement in dissolution rate of a drug supported by mean dissolution time (MDT), dissolution efficiency (DE), and relative dissolution rate (RDR). DE was observed as $61.63 \%$ and it was increased almost by 3.0 folds from SD8 ODT compared with conventional tablets. MDT for SD8 formulation was reduced to $8.79 \mathrm{~min}$, compared with conventional tablet (25.76 min). The relative dissolution rate (RDR) of optimized SD8 formulation was found to be 2.09. These results demonstrated that faster release of FPN from SD8 formulation compared to SD4, marketed and conventional tablets.

FTIR spectra showed no interaction between FPN and PEG, as same principal peaks present in pure drug and optimised SD8 formulation. The results of DSC studies revealed that, the sharp endothermic peak of drug was broadened in optimised SD8 formulation indicating FPN was molecularly dispersed in carrier. XRD studies showed the sharp intense peaks of drug indicating FPN was in crystalline state. The decrease or disappearance of peaks intensity in optimised SD8 formulation indicated, either there is a reduction in the crystallinity of FPN or it has undergone solid state transition to amorphous form.

Stability studies of the optimised SD8 formulation was performed at accelerated condition. No significant variation $(p>0.05)$ in concentration of drug was observed, when determined using paired t-test. Similarity index value of 89.59 indicated that the similarity in dissolution profile of freshly prepared and after storage of optimised SD8 formulation.

\section{CONCLUSIONS}

An attempt was made to enhance the solubility and dissolution of FPN using SDs of PEG. In vitro dissolution rate of formulations with PEG10000 manifested best 
results compared with formulations with PEG 8000. Significant improvement $(\mathrm{p}<0.05)$ in percent drug release $\left(\mathrm{Q}_{20}\right)$ was observed with SD8 compared with SD4, marketed and conventional tablets containing pure drug. Enhancement in dissolution of FPN was observed possibly due solubilisation of drug in hydrophilic carrier or change in the crystallinity of the drug. The DE and MDT of SD8 formulation were also significantly improved compared with conventional tablets. In conclusion, formulation of PEG solid dispersions in less carrier ratio could be an alternative technique for enhancing solubility, dissolution and thereby improving the absorption of poor water soluble drugs like FPN.

\section{ACKNOWLEDGEMENTS}

The authors would like to acknowledge the FDC Limited, Mumbai, India, Matrix Labs, Hyderabad, India and $\mathrm{CDH}$, Delhi, India for the gift samples. The authors also thank management, Max Institute of Pharmaceutical Sciences, Chaitanya College of Pharmacy Education and Research, National Institute of Pharmaceutical Education and Research- Hyderabad for providing facilities.

\section{REFERENCES}

Bhaskar D, Rama RT, Sateesh KV. Formulation and pharmacokinetics of gelucire solid dispersions of flurbiprofen. Drug Dev Ind Pharm. 2015;41(8):1254-62.

Bikiaris DN. Solid dispersions, part I: recent evolutions and future opportunities in manufacturing methods for dissolution rate enhancement of poorly water-soluble drugs. Expert Opin Drug Del. 2011;8(11):1501-1519.

Biswal S, Sahoo J, Murthy PN. Characterisation of gliclazidePEG 8000 solid dispersions.Trop J Pharm Res. 2009;8(5):417424.

Bolourchian N, Mahboobian MM, Dadashzadeh S. The effect of PEG molecular weights on dissolution behavior of simvastatin in solid dispersions. Iran J Pharm Res. 2013;12(Suppl):11-20.

Chauhan B, Shimpi S,Paradkar A. Preparation and evaluation of glibenclamide-polyglycolized glycerides solid dispersions with silicon dioxide by spray drying technique. Eur J Pharm Sci. 2005;26(2):219-230.

Chella N, Daravath B, Kumar D, Rama RT. Formulation and pharmacokinetic evaluation of polymeric dispersions containing valsartan. Eur J Drug Metab Pharmacokinet. 2016;41(5):517-26.
Christian L, Jennifer D. Improving drug solubility for oral delivery using solid dispersions. Eur J Pharm Biopharm. 2000;50(1):47-60.

Cwiertnia B. Effect of water soluble carrier on dissolution profiles of diclofenac sodium. Acta Pol Pharm. 2013;70(4):721726.

Dong HO, Young JP, Jun HK, Chul SY, Han GC. Physicochemical characterization and in vivo evaluation of flurbiprofen-loaded solid dispersion without crystalline change. Drug Deliv. 2011;18(1):46-53.

Dordunoo SK,Ford JL,Rubinstein MH.Preformulation studies on solid dispersions containing triamterene or temazepam in polyethylene glycols or gelucire 44/14 for liquid filling of hard gelatin capsules. Drug Dev Ind Pharm. 1991;17(12):1685-1713.

El-Maghraby GM, Elsergany RN. Fast disintegrating tablets of nisoldipine for intra-oral administration. Pharm Dev Technol. 2014;19(6):641-650.

Faisal W, O'Hora TR, O’Driscoll CM, Griffin BT. A novel lipid-based solid dispersion for enhancing oral bioavailability of lycopene-in vivo evaluation using a pig model. Int J Pharm. 2013;453(2):307-314.

Horter D, Dressman JB. Influence of physicochemical properties on dissolution of drugs in the gastrointestinal tract. Adv Drug Deliver Rev. 2001;46(1):75-87.

Mathews BR. Regulatory aspects of stability testing in Europe. Drug Dev Ind Pharm.1999;25(7):831-856.

Ozkan Y, Doganay N, Dikmen N, Isimer A. Enhanced release of solid dispersions of etodolac in polyethylene glycol. Farmaco. 2000;55(6-7):433-438.

Patil MP, Gaikwad NJ. Preparation and characterization of gliclazide-polyethylene glycol 4000 solid dispersions. Acta Pharmaceut. 2009;59(1):57-65.

Sinha S, Ali M, Baboota S. Solid dispersion as an approach for bioavailability enhancement of poorly water-soluble drug ritonavir. AAPS PharmSciTec. 2010;11(2):518-527.

Suresh B, Subash J, Basanth BE, Rajeshri D, Raju J, Prabhakar RV. Physicochemical characterization and dissolution enhancement of loratadine by solid dispersion technique. Korean J Chem Eng. 2013;30(1)238-244. 
Veerareddy PR, Vemula SK. Formulation, evaluation and pharmacokinetics of colon targeted pulsatile system of flurbiprofen. J Drug Target. 2012;20(8):703-714.
Vippagunta SR, Maul KA, Tallavajhala S, Grant JW. Solid state characterization of nifedipine solid dispersions. Int J Pharm. 2002;236(1-2):111-123.

Received for publication on $21^{\text {st }}$ September 2016 Accepted for publication on $26^{\text {th }}$ June 2017 